\title{
Do We Serve a Need?
}

\author{
John Somberga, b
}

The need for a journal covering general areas of cardiovascular research is questioned by some. There are a considerable number of journals that cover the cardiovascular area with more and more journals specializing in niche areas: heart failure, arrhythmias, nuclear medicine, electrocardiography, interventional cardiology, or atrial fibrillation; some of the areas that have dedicated journals. But does one only want to read articles in a particular area? Or is there a need to be informed of research in many areas of cardiology that then can cross-fertilize our thoughts, giving insights and approaches that can be applied to problems in different fields? Some of the most creative work in research is done by individuals with expertise outside of the primary area of interest. Diversity of articles is a strength for cardiology research. Diversity also serves one's varied interests. A singularly focused journal can be easily replaced by a monthly review of an index. No need to read a journal, just have a list of articles on subjects of interest to review.

There are also major journals that cover diverse areas of cardiology; Circulation, JAAC, the American J Cardiology. However, small studies, pilot studies, initial investigative reports may not be competitive for publication in these journals. There needs to be outlets for the publication of these kinds of papers. A place is needed for the publication of hypothesis generating reports that are not confirmed by current research. We need a forum for the publication of ideas that are not in vogue. There also needs to be a venue for articles that have inherent flaws but still present information worth discussing that may be the basis for further studies. Some of the most thorough, complete, and robust studies are repetitions and often present little new content. But new, and novel studies may be incomplete and can only be considered hypothesis generating. These studies also need to be published. Studies can be given editorial help to better present the author's ideas. Additionally, papers presenting novel ideas but that may not be acceptable to leading publications can be published in smaller journals questioning our scientific biases.

It is also well known that some of the most prestigious journals in the field engage in considerable editing of an investigator's presentation. Conclusions are modified, often understated, with the findings presented in hard-to-understand ver-

Manuscript submitted September 22, 2021, accepted September 24, 2021

Published online September 29, 2021

aCardiology \& Pharmacology, Rush University, Chicago, IL 60612, USA.

Email: John_Somberg@rush.edu

${ }^{b}$ Editor-in-Chief, Cardiology Research

doi: https://doi.org/10.14740/cr1324 biage or statistical formats difficult for the average clinician to understand. A smaller journal might be a venue for authors who want to avoid these pressures.

Cardiovascular research believes in letting authors present their conclusions, as controversial as they may be with as much support as the investigator can muster. The limitations section of the paper is where the investigator needs to reveal the flaws in their research, the lack of support for their conclusions, the deficiencies in the publication's methodology and where future studies are needed. The limitations section is where the reviewers and editors can be most helpful in encouraging the authors to reveal study weaknesses and the need for further investigation.

There is indeed a need for journals that cover a broad range of topics, that work with authors to present their finding as best they can and to point out the limitations of their methods and findings so they can be addressed in future studies. This is especially important for a journal with an international base, which brings to the research community ideas from many different societies, some with more sophisticated techniques and resources than others, but all with potentially useful insights.

\section{Acknowledgments}

None to declare.

\section{Financial Disclosure}

None to declare.

\section{Conflict of Interest}

None to declare.

\section{Data Availability}

The author declares that data supporting the findings of this study are available within the article. 